\title{
VARIABILIDAD EN CONTENIDO Y TIPOS DE ANTOCIANINAS EN GRANOS DE COLOR AZUL/MORADO DE POBLACIONES MEXICANAS DE MAÍZ
}

\author{
CONTENT AND TYPE VARIABILITY OF ANTHOCYANINS IN BLUE/PURPLE \\ COLORED GRAINS OF MEXICAN MAIZE POPULATIONS
}

\author{
Yolanda Salinas Moreno ${ }^{\star}$, Carolina García Salinas² ${ }^{2}$ Bulmaro Coutiño Estrada ${ }^{3}$ y Víctor A. Vidal Martínez ${ }^{4}$
}

\begin{abstract}
${ }^{1}$ Laboratorio de Calidad de Maíz. Campo Experimental "Valle de México". Instituto Nacional de Investigaciones Forestales, Agrícolas y Pecuarias (INIFAP). Km 13.5 carr. Los Reyes-Texcoco. 56250, Coatlinchan, Texcoco, Edo. de México. ${ }^{2}$ Departamento de Biotecnología e Ingeniería de Alimentos, Centro de BiotecnologíaFEMSA, Campus Monterrey, Tecnológico de Monterrey. Ave. Eugenio Garza Sada 2501 Sur. 64849, Monterrey, Nuevo León. ${ }^{3}$ Programa de Maíz. Campo Experimental Centro de Chiapas. INIFAP. 29140, Ocozocuautla, Chiapas. ${ }^{4}$ Campo Experimental Santiago Ixcuintla. (INIFAP). Apdo. Postal 100. Santiago. Ixcuintla, Nayarit. Tel. y fax 01(323)23-50710.
\end{abstract}

${ }^{*}$ Autor para correspondencia (yolasm@gmail.com)

\section{RESUMEN}

El objetivo de este trabajo es revisar y discutir la información disponible sobre contenido y caracterización de antocianinas en el grano de maíz (Zea mays L.), con énfasis en los maíces de razas mexicanas. El contenido y tipo de antocianinas en el grano de maíz varían de acuerdo con el color del grano y la concentración del pigmento en las distintas estructuras. Los granos de color rojo magenta concentran las antocianinas en el pericarpio y la capa de aleurona y poseen hasta 10 veces más antocianinas que los de grano azul/morado, cuyas antocianinas se concentran en la capa de aleurona. El perfil cromatográfico de antocianinas en el grano magenta revela la presencia de hasta 11 antocianinas que derivan de cianidina (73.3 a $75.7 \%)$, pelargonidina (8.3 a $9.3 \%$ ) y peonidina (16.0 a $17.5 \%)$. Los maíces de grano azul/morado presentan un perfil parecido al de grano magenta, con predominancia de derivados monoacilados de cianidina. Gran parte de la información publicada sobre la caracterización de antocianinas en el grano de este cereal se ha realizado con granos púrpura o magenta de origen Andino, que se destina para la preparación de extractos de antocianinas. Los trabajos sobre contenido de antocianinas en maíces mexicanos de grano azul o azul/ morado son reducidos, y aún más los orientados a la caracterización de sus antocianinas. Dado que en México este tipo de maíces se destinan a la elaboración de diversos alimentos tradicionales, conviene impulsar su investigación para determinar con mayor profundidad la variabilidad existente.

Palabras clave: Zea mays, razas mexicanas, flavonoides, granos de color.

\section{SUMMARY}

This work analyzes current information on the content and characterization of anthocyanins from maize (Zea mays L.) grains, focusing on studies related to Mexican maize races. Anthocyanin content and type vary depending on color and location of the pigment in the grain structures. Magenta colored maize grains concentrate anthocyanins in the pericarp and the aleurone layer; blue/purple maize grain concentrate pigments only in the aleurone layer. Anthocyanin content in magenta grains is up to 10 fold richer than blue/purple grains. Chromatographic anthocyanin profiles of magenta grain show the presence of 9 to 11 anthocyanins derivatives from cyanidin (73.3 to $75.7 \%$ ), pelargonidin (8.3 to $9.3 \%$ ) and peonidin (16.0 to $17.5 \%)$. Blue/purple maize grain analysis shows similar profiles to magenta grain, with a marked presence of mono acylated derivatives of cyanidin. Most published information on anthocyanin characterization in maize grains originates from Andean purple or magenta grains, which are mainly used for pigment extraction. Only few studies on anthocyanin content use Mexican blue or blue/purple maize grains, and even fewer show their anthocyanin profile. Blue/purple maize grains are preferably employed in Mexico for preparation of diverse traditional dishes, thus it is appropriate to encourage studies that deeply explore variability in anthocyanin content and type in these maize grains.

Index words: Zea mays, Mexican landraces, flavonoids, colored grains.

\section{INTRODUCCIÓN}

México es considerado como uno de los principales centros de origen del maíz (Zea mays L.). La diversidad que existe en el país sobre esta gramínea es muy amplia, y así lo demuestran las más de 59 razas de maíz descritas (Sánchez et al., 2000). En muchas de estas razas se presentan variantes de grano pigmentado con coloraciones que van desde el negro hasta el rosa pálido, con los colores rojo y azul/morado como los más comunes (Salinas, 2000; Com. pers.) ${ }^{1}$. Estas tonalidades de colores se deben a las antocianinas, uno de los principales grupos de pigmentos vegetales visibles al ojo humano (Kong et al. 2003). Los maíces pigmentados son producidos por agricultores de subsistencia, en suelos marginales y en pequeñas superficies, y la mayor parte de su producción es para autoconsumo. Sin embargo, en estados como Puebla y México la relación beneficio/costo de la producción de maíz azul es de 2.24, superior a la razón 1.57 del maíz comercial de grano blanco (Arellano et al., 2003; Keleman y Hellin, 2009). De esta manera, el maíz azul posee un papel importante como maíz especial.

'Salinas M Y (2000) Antocianinas en el grano de maíces criollos mexicanos. Tesis de Doctorado. Programa de Fisiología Vegetal, Colegio de Posgraduados. Montecillos, México. 101 p. 
Las antocianinas en el grano de maíz se acumulan predominantemente en el pericarpio, en la aleurona o en ambas estructuras (Salinas et al., 1999). Trabajos recientes han reportado la presencia marginal ( 0.07 a $0.08 \mathrm{mg} /$ grano $)$ de estos fenoles en el endospermo almidonoso y el embrión (Cui et al., 2012). La acumulación del pigmento en las estructuras del grano determina el posible uso de este tipo de maíces. Si el pigmento se concentra en la aleurona, el grano puede canalizarse al proceso de nixtamalización para la elaboración de productos con tonalidades azules, mientras que si se acumula en el pericarpio y en cantidad suficiente el grano pigmentado podría considerarse para la extracción de pigmentos (Salinas et al., 1999; Salinas, 2009).

Las antocianinas, además de ser colorantes inocuos para el consumo humano, poseen importantes actividades biológicas entre las que destacan sus capacidades antioxidantes y antimutagénicas (Lopez-Martinez et al., 2009; Zhao et al., 2009), anti-proliferativas del crecimiento de células cancerosas de diversos tipos (Jing et al., 2008). Es también reconocida la actividad anti-inflamatoria de las antocianinas ( $\mathrm{Li}$ et al., 2012; Zhu et al., 2013 ). Investigaciones recientes han atribuido a las antocianinas del grano de maíz acción protectora hacia las nefropatías que se desarrollan en pacientes con diabetes Tipo 2 (Li et al., 2012).

Las múltiples actividades biológicas de las antocianinas hacen que este grupo de flavonoides sea objeto de una activa investigación. De las diferentes antocianidinas o agliconas presentes en la mayoría de las frutas más comercializadas y consumidas por el hombre, la cianidina tiene un papel preponderante, tanto por su amplia distribución (De Pascual-Teresa y Sanchez-Ballesta, 2008) como por poseer un alto poder antioxidante (Rice-Evans et al., 1996). En los granos de maíz con coloraciones azul, morado, negro y magenta, la antocianidina (aglicona) predominante es cianidina (Salinas et al., 1999; De Pascual-Teresa et al., 2002; Aoki et al., 2002), ya que poco más de $73 \%$ de las antocianinas se derivan de ella (Aoki et al., 2002; Salinas et al., 2005); en granos azul/morado de razas como Chalqueño la antocianidina llega a constituir hasta 83.3 \% (Salinas, 2000; Com. pers. $)^{1}$.

Se ha publicado abundante información sobre el contenido, y particularmente la identificación y caracterización de las distintas antocianinas presentes en el grano de maíz Peruano. Sin embargo, la información de este tipo para maíces mexicanos de grano azul o azul/morado, que son predominantes en las razas del país, se halla dispersa y en ocasiones en documentos de circulación restringida, como tesis e informes de proyectos. En este trabajo de revisión se analiza la información publicada sobre las antocianinas identificadas en el grano de maíz en general, el contenido $\mathrm{y}$ tipo de dichos pigmentos en accesiones o poblaciones de razas mexicanas con grano de color azul/morado, y se establecen conclusiones y perspectivas sobre el tema.

\section{ANTOCIANINAS EN MAÍZ}

Las antocianinas representan los principales pigmentos solubles en agua, que son visibles al ojo humano. Aunque se pueden encontrar en cualquier parte de la planta, son mucho más frecuentes en flores y frutos, estructuras en las que contribuyen a los brillantes colores rojos, azules y morados de estos tejidos vegetales (Harborne, 1967). Pertenecen al grupo de los flavonoides, y su estructura básica es un núcleo de flavona que consta de dos anillos aromáticos unidos por una unidad de tres carbonos (Figura 1).

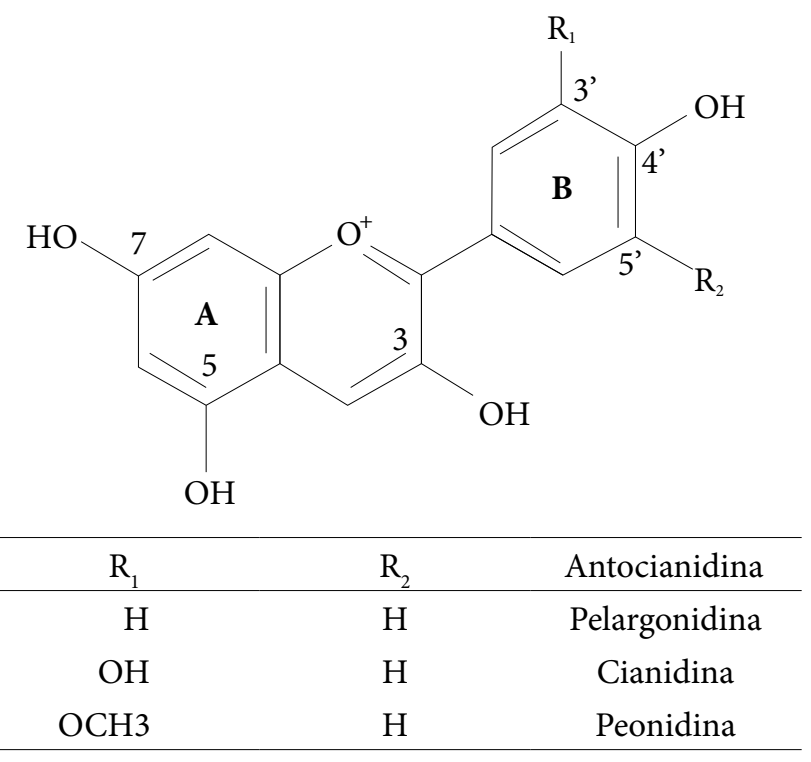

Figura 1. Estructura química de las antocianidinas más comunes en el grano de maíz (Zea mays L.). Adaptado de Harborne (1967).

Los sustituyentes en las posiciones 3', 4' y 5' del anillo B de la estructura determinan el tipo de antocianidina o aglicona. Las antocianidinas reportadas en el grano de maíz son cianidina y pelargonidina (Straus, 1959; Harborne y Gabazzi, 1969), aunque también se ha detectado la presencia de peonidina y malvidina (Caldwell y Peterson, 1992).

En maíz la mayoría de las antocianinas derivan de cianidina. Hay antocianinas cuya estructura está conformada por la antocianidina y un azúcar unido comúnmente a la posición 3' de la estructura de tres carbonos, y son las de tipo no acilado. Sin embargo, es posible que al azúcar se una un radical acilo proveniente de ácidos orgánicos, ya sean alifáticos o aromáticos; cuando esto ocurre se generan las antocianinas de tipo aciladas (De Pascual-Teresa y 
Sanchez-Ballesta, 2008). La presencia de grupos acilo en la molécula de antocianina le confiere estabilidad al pigmento ante condiciones extremas de $\mathrm{pH}$ y temperatura (Dougall et al., 1997). En las antocianinas no aciladas el principal azúcar es glucosa (Straus, 1959; Nakatani et al., 1979; Aoki et al., 2002), aunque algunos investigadores han identificado también al disacárido rutinosa (Abdel-Aal et al., 2006; Zilic et al., 2012).

En la planta de maíz las antocianinas están presentes en diferentes estructuras, como tallo, vaina, hojas e inflorescencias; en la mazorca se pueden encontrar en brácteas, raquis y grano. En el grano se ha reportado la presencia de antocianinas principalmente en el pericarpio, en la capa de aleurona, o en ambas estructuras (Salinas et al., 2005; Cui et al., 2012). La presencia de estos compuestos en el embrión o endospermo, sin incluir a la aleurona, es marginal (Cui et al., 2012).

El contenido de antocianinas totales en los granos de maíz puede variar en función del color del grano. En los maíces con tonalidades azul/morado, púrpura o magenta el contenido es mayor que en los granos de color rojo (Salinas et al., 1999; Lopez-Martinez et al., 2009; Zilic et al., 2012).

En el grano de maíz morado, púrpura o magenta, el más estudiado por su uso en la elaboración de extractos comerciales de pigmentos tipo antociano, los contenidos de antocianinas totales varían de acuerdo con las técnicas de extracción y del germoplasma utilizado. Salinas et al. (2005) analizaron cuatro muestras de grano de maíz magenta de distintas razas; el mayor contenido lo encontraron en el grano de la raza Arrocillo (1150 mg equivalentes de cianidina 3-glucósido (ECG)/kg de muestra seca), y el menor en la raza Cónico (540 mg ECG/kg MS).

En el grano completo de muestras de maíz de la misma tonalidad (magenta), Zhao et al. (2009) informaron contenidos de antocianinas de 2565 a $3045 \mathrm{mg}$ ECG/kg de materia seca (MS). Por su parte, Yang y Zhai (2010) reportaron valores de $558 \mathrm{mg}$ ECG/kg MS en el grano de maíz con la misma tonalidad. Estos valores son inferiores a los informados por Lopez-Martinez et al. (2009), quienes reportaron un contenido de $8500 \mathrm{mg}$ ECG/kg de MS para el maíz Peruano de Arequipa, que al parecer es el mismo tipo de maíz púrpura analizado por Montilla et al. (2011). En los trabajos antes mencionados, el pigmento en el grano se concentra en el pericarpio, estructura en la que el contenido llega a ser de hasta $14730 \mathrm{mg}$ ECG/kg de pericarpio (Salinas et al., 2005), mientras que en la aleurona el contenido es menor. Las diferencias entre los valores más altos y más bajos informados por los distintos autores, son de casi diez veces.
La identidad de cada antocianina de un extracto de antocianinas totales ha sido desde el principio una inquietud. Mediante técnicas cromatográficas de capa fina y de papel, Straus (1959) identificó a cianidina 3-glucósido, pelargonidina 3-monoglucósido y algunos derivados acilados, como las antocianinas predominantes en la variedad de maíz 'Negro mexicano dulce. Por su parte, Harborne y Self (1987) mediante el uso de cromatografía de líquidos de alta resolución (HPLC, por sus siglas en inglés), confirmaron la presencia de dos antocianinas aciladas [cianidina 3 - (6" malonilglucósido) y cianidina 3 dimalonilglucósido] en hojas coloreadas de maíz.

En fechas recientes se han publicado diversos trabajos sobre identificación de antocianinas en el grano de maíz mediante cromatografía líquida de alta resolución acoplada con espectrometría de masas (HPLC-MS). Esta herramienta analítica permite determinar la estructura química de cada antocianina y su identidad, sin tener que recurrir al uso de estándares comerciales (Aoki et al., 2002; Zilic et al., 2012).

El grano de maíz utilizado en la mayoría de estos trabajos ha sido el maíz morado, púrpura o magenta de la región andina (Bolivia y Perú). Los análisis se han efectuado directamente a partir del grano molido (Aoki et al., 2002; Montilla et al., 2011; Zilic et al., 2012), o en el extracto comercial del maíz peruano (De Pascual-Teresa et al., 2002). El perfil de antocianinas y su identidad fueron similares en ambos casos. Sin embargo, durante el proceso de obtención de los extractos comerciales de antocianinas de maíz pueden formarse algunos derivados, como etilmalonil glucósido de cianidina, pelargonidina y peonidina (De Pascual Teresa et al., 2002).

En el maíz peruano, con presencia de antocianinas predominantemente en el pericarpio, se han separado e identificado entre 8 y 11 antocianinas. Las encontradas en mayor proporción son: cianidina 3-glucósido, cianidina 3-(6"-malonil glucósido) y peonidina 3-glucósido. Otras antocianinas presentes en menor proporción son: peonidina 3-(6"-malonilglucósido), pelargonidina 3-glucósido, pelargonidina 3-(6"-malonilglucósido), además de dos isómeros, malonil y dimalonil (Aoki et al., 2002). Debe tenerse en cuenta que la proporción relativa de cada antocianina en granos del mismo color puede modificarse por efecto del genotipo (Salinas, 2000; Com. pers. ${ }^{1}$ ) y del ambiente de producción (Jing et al., 2007).

En la Figura 2 se muestran las estructuras químicas de las dos antocianinas más abundantes en el grano de color púrpura o magenta, que ha sido el grano más estudiado. Estas mismas antocianinas son también las más abundantes en los granos de color azul/morado (Zilic et al., 2012; Salinas 
et al., 2012a). Sin embargo, en los maíces con grano de color púrpura o magenta predomina la cianidina 3-glucósido sobre el derivado acilado (cianidina 3, 6" malonilglucósido), en tanto que en los de grano azul/morado con pigmento únicamente en la aleurona, la que domina es el derivado acilado (Salinas et al., 2012a).

En el Cuadro 1 se presenta información sobre las diferentes antocianinas identificadas en el grano de maíz púrpura o magenta. En dicho cuadro también se muestra la información sobre maíz de grano azul/morado estudiado por diversos autores.

Las antocianinas no aciladas identificadas en el grano de maíz son: cianidina 3-glucósido, pelargonidina 3-glucósido y peonidina 3-glucósido (Styles y Ceska, 1972). La primera es común en los maíces de grano azul/morado y magenta (Nakatani et al., 1979), y las dos restantes en maíces de grano rojo y magenta (Coe et al., 1955; Harborne y Gavazzi, 1969).

Las antocianinas aciladas con uno o más radicales acilo derivados de ácidos alifáticos (ácido málico, malónico o succínico) presentan en su estructura únicamente glucosa. Se han identificado en hojas de maíz coloreadas (Harborne y Self, 1987), en tanto que las aciladas con alguno de los cuatro ácidos cinámicos (p-cumárico, caféico, ferúlico o sinápico) poseen en su estructura glucosa y rutinosa. Las antocianinas identificadas en granos de maíz con color en la aleurona son: peonidina 3-(cafeilrutinósido)-5 glucósido,

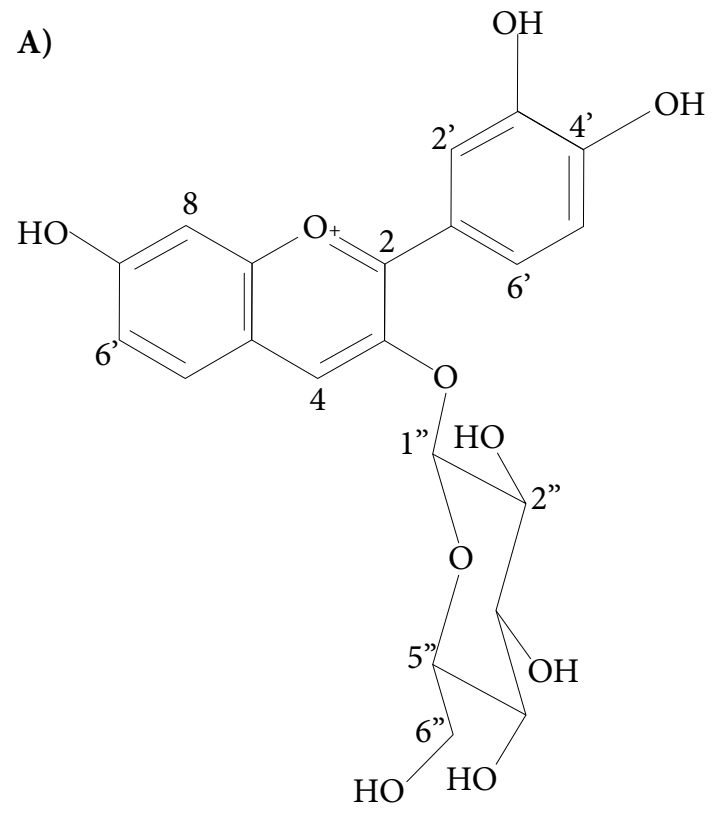

peonidina 3-(p-coumarilrutinósido)-5 glucósido y malvidina 3-(cafeilrutinósido)-5 glucósido (Caldwell y Peterson, 1992; Gonzalez-Manzano et al., 2008).

\section{Antocianinas en el grano de maíz de poblaciones mexicanas con tonalidades azul o azul/morado}

De acuerdo con Wellhausen et al. (1951), en la mayoría de las razas mexicanas de maíz se presentan variantes con coloraciones de grano desde rosa hasta morado intenso o negro, que son debidas a las antocianinas. Los colores de grano con presencia de antocianinas más comunes en los maíces nativos mexicanos son azul/morado y rojo cereza (Salinas et al., 1999). El contenido de antocianinas en el grano de estas poblaciones puede variar enormemente. Espinosa (2003; Com. pers.) $)^{2}$ determinó el contenido de antocianinas en 10 razas de maíz de grano azul/morado proporcionadas por el banco de germoplasma del Instituto Nacional de Investigaciones Forestales, Agrícolas y Pecuarias (INIFAP), reportó una variabilidad de 51.92 a 144.34 $\mathrm{mg}$ de pelargonidina clorada/kg de muestra seca (MS). La raza con mayor contenido fue Cónico y la de menor fue Chiquito. El número de poblaciones analizadas dentro de cada raza fue limitado (1 a 2). Salinas (2009) encontró valores parecidos a los de Espinosa (2003; Com. pers.) ${ }^{2}$ en accesiones de maíces de grano azul de 20 razas, cada una

${ }^{2}$ Espinosa G B M (2003) Antocianinas en maíces de grano pigmentado (Zea mays L.) y medición de su actividad antioxidante. Tesis de Licenciatura. Departamento de Ingeniería Agroindustrial, Universidad Autónoma Chapingo. Chapingo, Méx. 101 p.

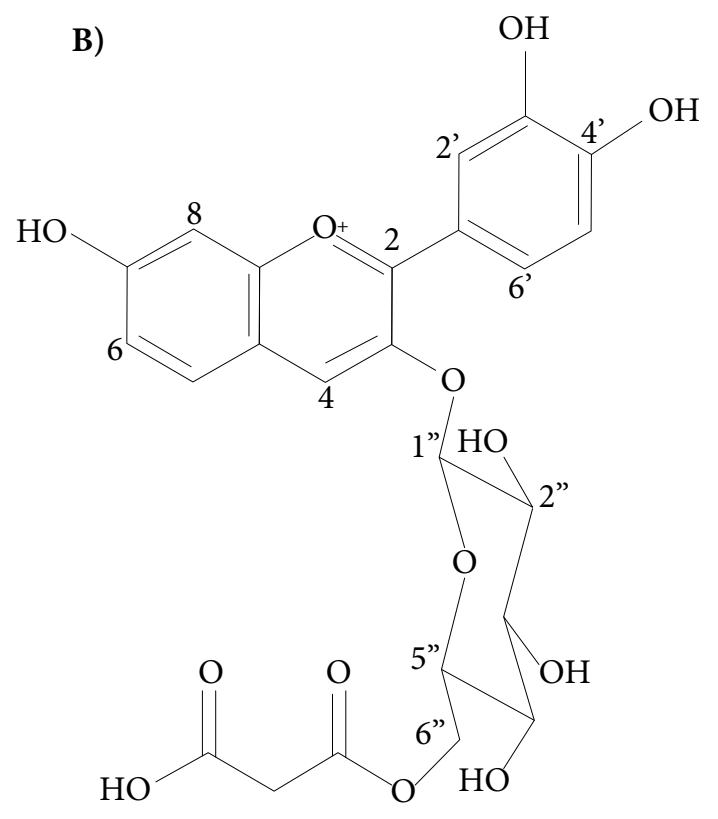

Figura 2. Estructuras químicas de las antocianinas más abundantes en el grano de maíz de color azul/morado. A) cianidina 3-glucósido, B) cianidina 3-(6"-malonilglucósido). (Adaptado de Fossen et al., 2001). 
Cuadro 1. Color de grano, ubicación del pigmento y antocianinas identificadas en granos de maíz (Zea mays L.) con diferentes coloraciones.

\begin{tabular}{|c|c|c|c|}
\hline Color de grano & $\begin{array}{l}\text { Ubicación del } \\
\text { pigmento }\end{array}$ & Antocianina identificada & Referencia \\
\hline $\begin{array}{l}\text { Morado, Azul / } \\
\text { morado }\end{array}$ & $\begin{array}{c}\text { Aleurona, Pericarpio } \\
\text { y aleurona }\end{array}$ & $\begin{array}{l}\text { Cianidina- 3-glucósido, Pelargonidina } \\
\text {-3-glucósido }\end{array}$ & $\begin{array}{l}\text { Styles y Ceska, (1972), Nakatani et al. } \\
\text { (1979), Salinas et al. (1999), De Pascual- } \\
\text { Teresa et al. ( 2002) }{ }^{\dagger} \text {, Aoki et al. (2002), } \\
\text { Abdel-Aal et al. (2006), Cortes et al. } \\
\text { (2006), Pedreschi y Cisneros-Zevallos } \\
\text { (2007), Montilla et al. (2011), Zilic et al. } \\
\text { (2012), Salinas et al. (2012a) }\end{array}$ \\
\hline Azul & Aleurona & Cianidina- 3-rutinósido & Abdel-Aal et al. (2006), Zilic et al. (2012) \\
\hline Morado $^{\dagger \dagger}$ & $\begin{array}{l}\text { Pericarpio y } \\
\text { aleurona }\end{array}$ & Peonidina- 3-glucósido & $\begin{array}{l}\text { Aoki et al. (2002), Abdel-Aal et al. (2006), } \\
\text { Pedreschi y Cisneros-Zevallos (2007), } \\
\text { Montilla et al. (2011), Zilic et al. (2012) }\end{array}$ \\
\hline Azul/morado & Aleurona & Peonidina- 3-glucósido & Zilic et al. (2012), Salinas et al. (2012a) \\
\hline Morado & Aleurona & Cianidina-3-(6”-malonilglucósido) & Aoki et al. (2002) \\
\hline Azul/morado & Aleurona & Cianidina-3-(6”-malonilglucósido) & $\begin{array}{c}\text { Montilla et al. (2011), Zilic et al. (2012), } \\
\text { Salinas et al. (2012a) }\end{array}$ \\
\hline Azul/morado & Aleurona & Cianidina succinil glucósido & $\begin{array}{c}\text { Abdel-Aal et al. (2006), Salinas et al. } \\
(2012 \mathrm{a})\end{array}$ \\
\hline Morado $^{\dagger \dagger}$ & $\begin{array}{l}\text { Pericarpio y } \\
\text { aleurona }\end{array}$ & Pelargonidina-3- (6"-malonilglucósido) & $\begin{array}{c}\text { Aoki et al. (2002), Montilla et al. (2011), } \\
\text { Zilic et al. (2012) }\end{array}$ \\
\hline Azul/morado & Aleurona & & Zilic et al. (2012), Salinas et al. (2012a) \\
\hline Morado $^{\dagger \dagger}$ & $\begin{array}{l}\text { Pericarpio y } \\
\text { aleurona }\end{array}$ & Peonidina-3-(6"-malonilglucósido) & $\begin{array}{c}\text { Aoki et al. (2002), Montilla et al. (2011), } \\
\text { Zilic et al. (2012) }\end{array}$ \\
\hline Azul/morado & Aleurona & & Zilic et al. (2012), Salinas et al. (2012a) \\
\hline Morado $^{\dagger \dagger}$ & $\begin{array}{l}\text { Pericarpio y } \\
\text { aleurona }\end{array}$ & Cianidina 3-(dimalonilglucósido) & $\begin{array}{c}\text { Aoki et al. (2002), Montilla et al. (2011), } \\
\text { Zilic et al. (2012) }\end{array}$ \\
\hline Azul/morado & Aleurona & & Zilic et al. (2012), Salinas et al. (2012a) \\
\hline Morado $^{\dagger \dagger}$ & $\begin{array}{l}\text { Pericarpio y } \\
\text { aleurona }\end{array}$ & $\begin{array}{c}\text { Pelargonidina 3-(dimalonilglucósido) } \\
\text { y Peonidina 3-(dimalonilglucósido) }\end{array}$ & $\begin{array}{c}\text { Aoki et al. (2002), Montilla et al. (2011), } \\
\text { Zilic et al. (2012) }\end{array}$ \\
\hline
\end{tabular}

${ }^{\dagger}$ Se analizó un extracto comercial de maíz morado; ${ }^{\dagger \dagger}$ Se refiere al grano magenta de la región Andina.

representada por una accesión (Figura 3).

Por su parte, Robles (2004; Com. pers.) $)^{3}$ cuantificó el contenido de antocianinas totales en el grano de una accesión por cada una de las razas Chalqueño, Bolita y Cónico, las tres de color de grano azul/morado (Figura 4). Los valores que obtuvo fueron: 417.4, 441.0 y $296.9 \mathrm{mg}$ equivalentes de

${ }^{3}$ Robles R R R (2004) Actividad antioxidante de masa y tortilla de maíces pigmentados. Tesis de Licenciatura. Departamento de Ingeniería Agroindustrial, Universidad Autónoma Chapingo. Chapingo, Méx. 67 p. pelargonidina clorada/kg MS, respectivamente.

En algunos trabajos de cuantificación de antocianinas en muestras de maíces nativos no se indica la raza a la que pertenecen las colectas o accesiones analizadas. Tal es el caso del reporte de Espinosa et al. (2009), quienes analizaron dos colectas de maíz nativo con grano azul y morado obtenidas en Tlaxcala e Hidalgo, respectivamente. Los contenidos de antocianinas totales reportados por estos autores fueron de 371.7 y $154.0 \mathrm{mg}$ ECG/kg MS, para las 


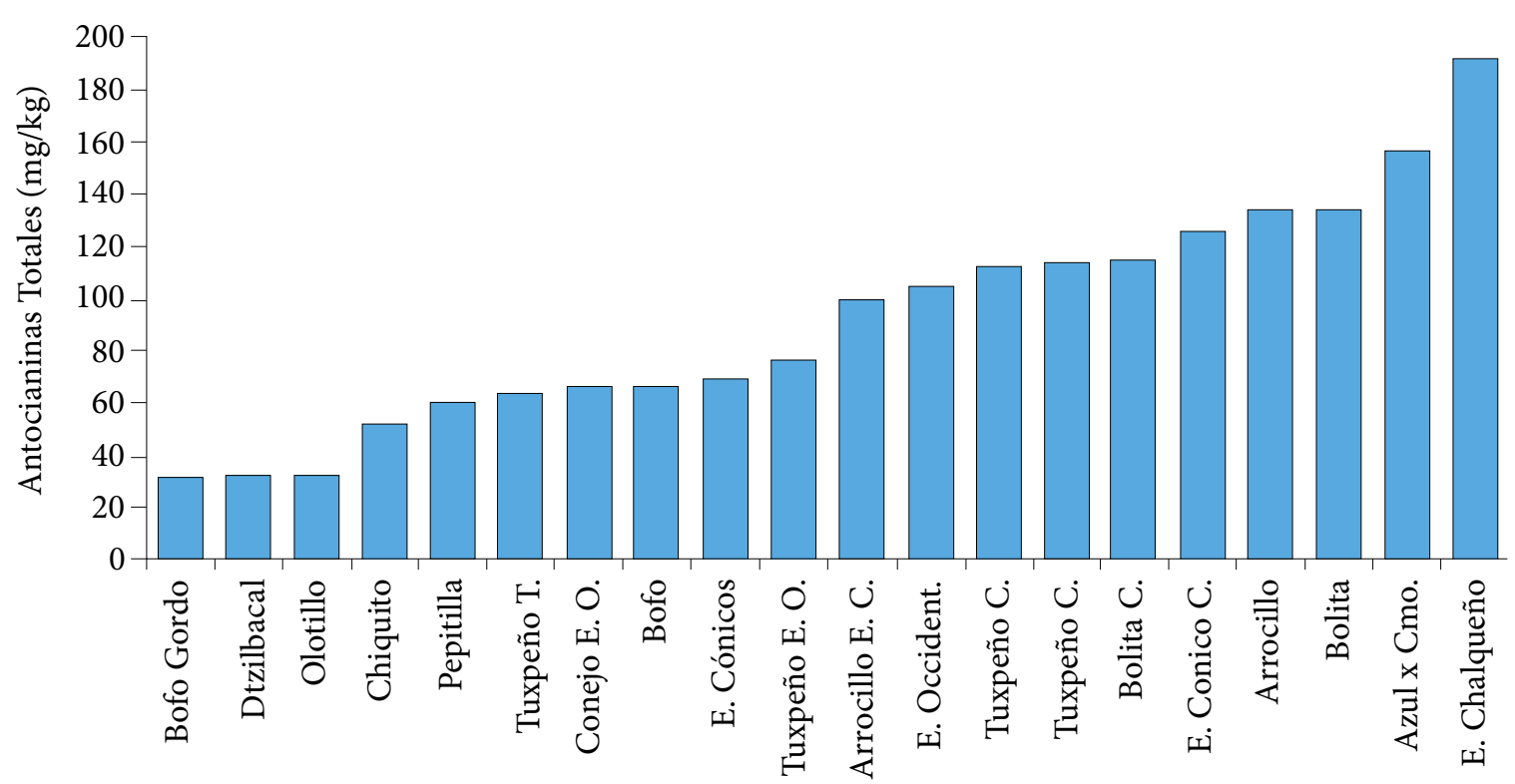

Figura 3. Contenido de antocianinas totales en grano de maíz azul de 20 razas (Adaptado de Salinas, 2009). Tuxpeño T: Tuxpeño Tepecintle; Conejo E. O.: Conejo Elotes Occidentales; E. Cónicos: Elotes Cónicos; Tuxpeño E. O.: Tuxpeño Elotes Occidentales; Arrocillo E. C.: Arrocillo Elotes Cónicos; E. Occident: Elotes Occidentales; Tuxpeño C.: Tuxpeño Cónico; Bolita C.: Bolita Cónico; E. Cónico C.: Elotes Cónicos Cónico; Azul x Crno: Azul x Cristalino de Chihuahua; E. Chalqueño: Elotes Chalqueños.
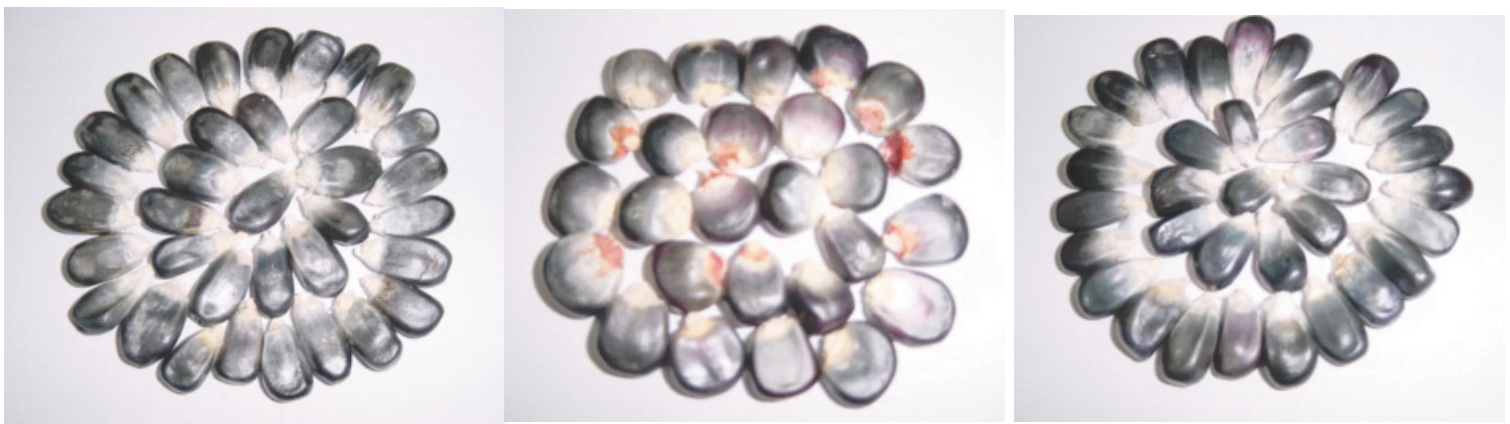

Figura 4. Granos de color azul morado en accesiones de tres razas de maíz, Chalqueño (a), Bolita (b) y Cónico (c), analizadas por Robles (2004, Com. pers.) ${ }^{3}$.

muestras de grano azul y morado, en ese orden. Flores (2011; Com. pers.) $)^{4}$ determinó el contenido de antocianinas en 12 poblaciones de maíz con grano azul y negro provenientes de la región de Huaquechula, Puebla, en sitios de colecta ubicados a alturas entre 1400 y $1700 \mathrm{msnm}$. La variabilidad observada entre estas poblaciones fue de 790 a $1690 \mathrm{mg} / \mathrm{kg}$ de muestra, pero no mencionaron el estándar usado para calcular la concentración de antocianinas, y tampoco especificaron si los resultados están en base seca o húmeda, lo que dificulta su comparación con otros trabajos.

${ }^{4}$ Flores P V (2011) Rendimiento de grano y contenido de antocianinas en poblaciones de maíz de la región de Huaqhechula, Puebla. Tesis de Maestría. Colegio de Posgraduados, campus Puebla. 50 p.
En razas adaptadas a altitudes elevadas tales como Chalqueño y Elotes Cónicos se ha observado un contenido mayor de antocianinas (Salinas, 2010; Com. pers.) $)^{5}$ que en razas endémicas de alturas menos elevadas como Olotillo. En esta raza (Olotillo), Salinas et al. (2012b) analizaron el contenido de antocianinas totales en 20 accesiones de grano azul/morado obtenidas en localidades de Chiapas situadas a alturas no mayores a $1500 \mathrm{msnm}$, y reportaron valores que variaron desde 276.8 hasta $904.0 \mathrm{mg}$ ECG/kg MS. En este mismo trabajo se informa del contenido de antocianinas en

${ }^{5}$ Salinas M Y, J Soria R, E Espinos T (2010) Aprovechamiento y distribución de maíz azul en el Estado de México. Folleto Técnico No. 42. CEVAMEX-INIFAP. $50 \mathrm{p}$ 
otras razas comunes en el Estado de Chiapas, como Olotón, Tehua, Zapalote Grande, Tuxpeño, Tepecintle y Vandeño, en las cuales se analizó un número reducido de accesiones. En el Cuadro 2 se muestra la información sobre contenido de antocianinas en accesiones de distintas razas de maíz de México.

En seis accesiones de Chalqueño la variabilidad reportada por Salinas et al. (2012a) fue de 579.4 a $1046.1 \mathrm{mg}$ ECG/ kg MS; para Elotes Cónicos fue de 997.8 a 1332.2 mg ECG/ kg MS. Tanto Chalqueño como Elotes Cónicos son razas adaptadas a alturas superiores a los $2000 \mathrm{msnm}$, con condiciones ambientales donde prevalecen altas luminosidades y bajas temperaturas nocturnas que favorecen la síntesis y acumulación de antocianinas. La bajas temperaturas reducen los niveles del sistema enzimático que inactiva la síntesis de la enzima L-fenilalanina amonio-liasa (PAL) que es indispensable para la síntesis de flavonoides (Faragher, 1983).

En la raza Bolita la variabilidad reportada por Salinas et al. (2012a) en seis accesiones analizadas fue de 304.1 a 528.0 $\mathrm{mg}$ ECG $/ \mathrm{kg}$ MS. Bolita es una raza adaptada a ambientes de elevaciones medias, entre 900 a $1500 \mathrm{msnm}$ (Wellhausen et al., 1951), y su grano es de dureza intermedia a dura.

Tabloncillo es una raza de maíz adaptada a alturas medianas (1500 msnm), con distribución amplia en el Estado de Nayarit. En esta raza se presentan variantes de grano azul/ morado, con dureza entre intermedia y dura. Los contenidos de antocianinas totales informados por Salinas (2006; Com. pers. $)^{6}$ en ocho accesiones analizadas, fueron desde 199.2 hasta $344.6 \mathrm{mg}$ ECG/kg MS.

En general, los contenidos de antocianinas en accesiones de razas adaptadas a altitudes medianas son parecidos a los informados por otros autores en muestras de grano con tonalidades azules o azul/moradas. Por ejemplo, Abdel-Aal et al. (2006) señalaron un contenido de $196.7 \mathrm{mg}$ ECG $/ \mathrm{kg}$ de MS para una muestra de grano azul, en tanto que De la

${ }^{6}$ Salinas M Y (2006) Informe técnico del análisis de calidad Nixtamaleratortillera en 37 muestras del Noroeste de México.

\section{Cuadro 2. Contenido de antocianinas en el grano de 17 razas mexicanas de maíz.}

\begin{tabular}{|c|c|c|c|c|c|}
\hline Raza de maíz & $\begin{array}{c}\text { Núm. de colectas } \\
\text { analizadas }\end{array}$ & Color de grano & $\begin{array}{l}\text { Estructura del gra- } \\
\text { no analizada }\end{array}$ & $\begin{array}{c}\text { Contenido de anto- } \\
\text { cianinas }\end{array}$ & Referencia \\
\hline Chalqueño & 1 & Azul/morado & $\begin{array}{c}\text { Grano sin germen } \\
(\mathrm{A})^{\dagger}\end{array}$ & $417.4^{\dagger \dagger}$ & $\begin{array}{l}\text { Robles (2004; Com. } \\
\text { pers.) }\end{array}$ \\
\hline Cónico & 1 & “ $а$ & “ “ & $441.0^{\dagger \dagger}$ & “ $а$ \\
\hline Bolita & 1 & “ & “ & $296.9^{\dagger \dagger}$ & “ \\
\hline NI & 1 & Azul & Grano entero (A) & $371.7^{9}$ & Espinosa et al. (2009) \\
\hline NI & 1 & Morado & “ “ & $154.0^{9}$ & Espinosa et al. (2009) \\
\hline NI & 12 & Azul y negro & Grano entero (A) & $790-1690^{99}$ & $\begin{array}{c}\text { Flores (2011; Com. } \\
\text { Pers })^{4}\end{array}$ \\
\hline Olotillo & 20 & Azul/morado & $\begin{array}{c}\text { Grano sin germen } \\
\text { (A) }\end{array}$ & $276.8-904.0^{9}$ & Salinas et al. (2012b) \\
\hline Olotón & 1 & “ & 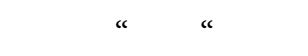 & $294.1^{9}$ & “ \\
\hline Tehua & 3 & “ & “ & $213.6-401.9^{9}$ & “ \\
\hline Zapalote Grande & 3 & “ & “ & $519.9-591.1^{9}$ & “ \\
\hline Tuxpeño & 4 & “ & “ & 216.8 - 367.9 & “ \\
\hline Tepecintle & 1 & “ & “ & $512.0^{9}$ & “ \\
\hline Vandeño & 2 & “ & “ & $485.2-657.3$ & “ \\
\hline Chalqueño & 6 & “ & “ & $579.4-1046.9$ & Salinas et al. (2012a) \\
\hline Elotes Cónicos & 6 & “ & “ & $997.8-1332.2^{9}$ & « « \\
\hline Bolita & 6 & “ “ & “ & $304.1-528.0$ & “ \\
\hline Tabloncillo & 8 & “ & “ & $199.2-344.6^{9}$ & $\begin{array}{l}\text { Salinas (2006; Com. } \\
\text { pers. })^{6}\end{array}$ \\
\hline
\end{tabular}


Parra et al. (2007) reportaron un valor de $368.7 \mathrm{mg}$ ECG $/ \mathrm{kg}$ de MS para una muestra de grano azul. Por su parte, MoraRochin et al. (2011) informaron un contenido de $306.9 \mathrm{mg}$ ECG/kg de MS.

En todos estos casos estos valores son superiores a los reportados por Espinosa (2003; Com. Pers) $)^{2}$, lo que posiblemente se deba a que el contenido de antocianinas totales en este último haya sido expresado en equivalentes de pelargonidina clorada. Este es un estándar no muy adecuado para expresar el contenido de antocianinas en maíces de grano azul o azul/morado, en los que la mayoría de sus antocianinas son derivadas de cianidina (Salinas et al., 2012a), pero la pelargonidina clorada es mucho más económica que la cianidina 3-glucósido. Por tanto, el estándar usado para elaborar la curva patrón necesaria para realizar los cálculos, es un factor adicional a los ya mencionados en párrafos previos, que puede afectar la variabilidad de los valores reportados en maíces de color azul/morado.

En todos los casos, los valores de antocianinas totales reportados en muestras de grano azul o azul/morado son considerablemente inferiores a los de maíces andinos con color de grano púrpura o magenta (Aoki et al. 2002; Zhao et al., 2009; Montilla et al., 2011), que son los que se destinan a la obtención de extractos comerciales de antocianinas.

\section{Caracterización de las antocianinas del grano de maíz en razas mexicanas con tonalidades azul o azul/morado}

Un aspecto poco estudiado en los maíces de grano azul o azul/morado es lo relacionado con la identificación y caracterización de sus antocianinas, debido posiblemente a que estudios de este tipo requieren de equipos costosos, de no fácil acceso y manejo como el HPLC-MS. La identidad de cada antocianina y la cuantificación del porcentaje relativo en la mezcla permiten orientar mejor el uso específico para cada maíz, y estimar de alguna manera la estabilidad de sus antocianinas ante determinadas condiciones de proceso.

En muestras de grano con tonalidades azul/moradas, pero no de razas mexicanas de maíz, se ha reportado un perfil de antocianinas parecido al del maíz Peruano de color púrpura o magenta (Zilic et al., 2012). Las principales diferencias entre este color de grano, con pigmento en el pericarpio y en la capa de aleurona, y los granos de tonos azul/ morados con pigmento únicamente en la capa de aleurona, se encuentran en la proporción relativa entre antocianinas no aciladas y aciladas. El maíz con grano púrpura o magenta tiene una mayor proporción de antocianinas no aciladas/ aciladas que el grano de color azul/morado. Esta diferencia determina la estabilidad del pigmento a $\mathrm{pH}$ y temperatura. Una mayor proporción de antocianinas aciladas puede significar mayor estabilidad (Bakowzka-Barezak, 2005).
En accesiones de grano azul/morado de las razas Chalqueño, Elotes Cónicos y Bolita, Salinas et al. (2012a) encontraron un perfil similar de antocianinas entre las accesiones de las tres razas, particularmente entre Chalqueño y Elotes Cónicos. En las seis accesiones de Bolita analizadas no se observó derivado alguno de peonidina, que sí estuvo presente en las accesiones de las otras dos razas. En el perfil de agliconas de Bolita no se halló peonidina, y su proporción de pelargonidina fue mayor que la registrada en las accesiones de las razas Chalqueño y Elotes Cónicos. Estas diferencias entre razas con un mismo color de grano influyen en el color de los productos finales que se elaboran a partir de los maíces.

El efecto del ambiente de producción sobre el contenido y la proporción relativa de cada antocianina en variantes de razas mexicanas con grano azul/morado prácticamente no se ha estudiado, aunque en otros cultivos se ha demostrado una elevada interacción entre ambiente y contenido de fenoles, particularmente en Hibiscus sabdariffa L. cuyo contenido de antocianinas en los cálices se duplicó por efecto de localidad (Juliani et al., 2009). De los pocos trabajos sobre este tema está el desarrollado por Salinas (2000; Com pers) ${ }^{1}$, quien indicó un efecto del ambiente sobre la proporción relativa de antocianinas, pero sin modificar el perfil. Jing et al. (2007) reportaron resultados similares, además de un efecto importante del ambiente en el contenido de antocianinas en el olote del maíz púrpura Peruano cultivado en diferentes localidades del Perú.

\section{CONCLUSIONES Y PERSPECTIVAS}

El contenido y tipo de antocianinas en el grano de maíz varían de acuerdo con el color del grano y la concentración del pigmento en las distintas estructuras. Los granos de color rojo magenta concentran las antocianinas en el pericarpio y en la capa de aleurona, y poseen hasta 10 veces más antocianinas que los de grano azul/morado cuyas antocianinas se concentran solamente en la capa de aleurona. El perfil cromatográfico de antocianinas en el grano magenta revela la presencia de hasta 11 antocianinas derivadas de cianidina (73.3 a $75.7 \%)$, pelargonidina (8.3 a $9.3 \%$ ) y peonidina (16.0 a $17.5 \%$ ). Los maíces de grano azul/morado presentan un perfil parecido al de grano magenta, con predominancia de derivados monoacilados de cianidina.

El gran número de estudios sobre contenido y caracterización de antocianinas en el grano magenta de origen Andino, cuyo uso principal es la obtención de extractos de antocianinas para emplearse en alimentos, contrasta con los pocos trabajos sobre la caracterización de antocianinas en granos de color azul/morado que en México son destinados a la elaboración de alimentos tradicionales. 
Es evidente la necesidad de ampliar la información sobre las razas mexicanas de maíz con variantes de grano azul/ morado. Se requiere fortalecer los grupos interdisciplinarios de trabajo con la participación de expertos en la clasificación de las colectas o accesiones, para que se integre la información sobre clasificación racial y se pueda conocer el avance en la caracterización de este germoplasma.

Dado el extenso uso tradicional del maíz azul en México y su potencial en mercados especializados, la información científica en las áreas de diversidad genética, mejoramiento, calidad y nutrición, al igual que en cadenas de valor, es necesaria por su contribución para la toma de decisiones y diseño de políticas públicas.

\section{AGRADECIMIENTOS}

Al programa "MASAGRO: Modernización sustentable de la agricultura tradicional", de SAGARPA-CIMMYT, por su apoyo para la publicación de este manuscrito, y a N. Palacios Rojas por su revisión técnica inicial.

\section{BIBLIOGRAFÍA}

Abdel-Aal E-S M P, J C Young, I Rabalski (2006) Anthocyanin composition in black, blue, pink, purple and red cereal grains. J. Agric. Food Chem. 54:4696-4704.

Aoki H, N Kuze, Y Kato (2002) Anthocyanins isolated from purple corn (Zea mays L.). Foods \& Food Ingred. J. Jpn. 199: 41-45.

Arellano V J L, C Tut Couoh, A M Ramírez, Y Salinas M, O R Taboada G (2003) Maíz azul de los Valles Altos de México. I: Rendimiento de grano y caracteres agronómicos. Rev. Fitotec. Mex. 26:101-107.

Bakowska-Barezak A (2005) Acylated anthocyanins, as stable natural food colorants- A review. Pol. J. Food Nutr. Sci. 14/55:107-116.

Caldwell E E O, P A Peterson (1992) HPLC Identification of anthocyanins in maize endosperm. Maize Genet. Coop. Newslett. 66:2.

Coe E H (1955) Anthocynin synthesis in maize, the interaction of $A_{2}$ and $\mathrm{P}$ in leucoanthocyanin accumulation. Genetics 40:568.

Kong G-M, L-S Chia, N-K Goh, T-F Chia, R Brouillard (2003) Analysis and biological activities of anthocyanins. Phytochemistry 64:923-933.

Cortes G A, Y Salinas-Moreno, E SanMartin-Martinez, F MartinezBustos (2006) Stability of anthocyanins of blue maize (Zea mays L.) after nixtamalization of separated pericarp-germ tip cap and endosperm fractions. J. Cereal Sci. 43:57-62.

Cui L, G Rongqi, D Shuting, J Zhang, L Peng, H Zhang, J Meng, D Shi (2012) Effects of ear shading on the anthocyanin contents and quality of kernels in various genotypes of maize. Aust. J. Crop Sci. 4:704-710

De la Parra C, S O Serna-Saldivar, R H Liu (2008) Effect of processing on the phytochemical profiles and antioxidant activity of corn for production of masa, tortillas, and tortilla chips. J. Agric. Food Chem. 55:4177-4183.

De Pascual-Teresa S, C Santos-Buelga, J C Rivas-Gonzalo (2002) LCMS analysis of anthocyanins from purple corn cob. J. Sci. Food Agric. 82:1003-1006.

De Pascual-Teresa S, M T Sanchez-Ballesta (2008) Anthocyanins: from plant to health. Phytochem. Rev. 7:281-299.

Dougall D K, D C Baker, E Gakh, M Redus (1997) Biosynthesis and stability of monoacylated anthocyanins. Food Technol. 15:69-71.

Espinosa T E, M C Mendoza C, F Castillo G, J Ortiz C, A Delgado A, A Carrillo $S$ (2009) Acumulación de Antocianinas en pericarpio y aleurona del grano y sus efectos genéticos en poblaciones criollas de maíz pigmentado. Rev. Fitotec. Mex. 32:303-309.

Faragher J D (1983) Temperature regulation of anthocyanin accumulation. J. Exp. Bot. 34:1291-1298.

Fossen T, R Slimestad, $\varnothing$ M Andersen (2001) Anthocyanins from maize (Zea mays) and reed canarygrass (Phalaris arundinacea). J. Agric. Food Chem. 49:2318-2321.

Gonzalez-Manzano S, J J Perez-Alonso, Y Salinas-Moreno, N Mateus, A M S Silva, V de Freitas, C Santos-Buelga (2008) Flavanolanthocyanin pigments in corn: NMR characterization and presence in different purple corn varieties. J. Food Comp. Anal. 21:521-526.

Harborne J B (1967) The anthocyanin pigments. In: Comparative biochemistry of the flavonoids. Academic Press. New York, London.

Harborne J B, G Gavazzi (1969) Effect of Pr and pr alleles on anthocyanin biosynthesis in Zea mays. Phytochemistry 8:999-1001.

Harborne J B, R Self (1987) Malonated cyanidin 3 glucosides in Zea mays and other grasses. Phytochemistry 26:2417-2418.

Jing P, J A Bomser, S J Schwartz, J He, B A Magnuson, M M Giusti (2008) Structure-function relationships of anthocyanins from various anthocyanin-rich extracts on the inhibition of colon cancer cell growth. J. Agric. Food Chem. 56:9391-9398.

Jing P, V Noriega, S J Schwartz, M M Giusti (2007) Effects of growing conditions on purple corn cob (Zea mays L.) anthocyanins. J. Agric. Food Chem. 55:8625-8629.

Juliani H R, C R Welch, Q Wu, B Diouf, D Malainy, J E Simon (2009) Chemistry and quality of Hibiscus (Hibiscus sabdariffa) for developing the natural-product industry in Senegal. J. Food Sci. 74:S113-S121.

Keleman A, J Hellin (2009) Specialty maize varieties in Mexico: A case study in market-driven agro-biodiversity conservation. J. Lat. Am. Geogr. 8:147-174.

Li J, S S Lim, J Y Lee, J K Kim, S W Kang, J L Kim, Y H Kang (2012) Purple corn anthocyanins dampened high-glucose-induced mesangial fibrosis and inflammation: possible renoprotective role in diabetic nephropathy. J. Nutr. Biochem. 23:320-331

Lopez-Martinez L X, R M Oliart-Ros, G Valerio-Alfaro, C H Lee, K L Parkin, S H Garcia (2009) Antioxidant activity, phenolic compounds and anthocyanins content of eighteen strains of Mexican maize. LWT-Food Sci. Technol. 42:1187-1192.

Montilla C E, S Hillebrand, A Antezana, P Winterhalter (2011) Soluble and bound phenolic compounds in different Bolivian purple corn (Zea mays L.) cultivars. J. Agric. Food Chem. 59:70687074 .

Mora-Rochin S, J A Gutiérrez-Uribe, S O Serna-Saldivar, P SánchezPeña, C Reyes-Moreno, J Milán-Carrillo (2010) Phenolic content and antioxidant activity of tortillas produced from pigmented maize processed by conventional nixtamalization or extrusion cooking. J. Cereal Sci. 52:502-508.

Nakatani N, H Fukuda, H Fuwa (1979) Major anthocyanin of Bolivian purple corn (Zea mays L.). Agric. Biol. Chem. 43:389-391.

Pedreschi R, L Cisneros-Zevallos (2007) Phenolic profiles of Andean purple corn (Zea mays L.). Food Chem. 100:956-963.

Rice-Evans C A, N J Miller, G Paganga (1996) Structure antioxidant activity relationships of flavonoids and phenolic acids. Free Radical Biol. Med. 20:933-956.

Salinas M Y (2009) Uso de maíces con pigmento tipo antociano. En: Temas Selectos de la Cadena Maíz-Tortilla: Un Enfoque Multidisciplinario. A P De Teresa-Ochoa, G Viniegra-González (comps). Universidad Autónoma Metropolitana. pp:177-202.

Salinas M Y, F J Cruz Ch, S A Díaz O, F Castillo G (2012b) Granos de maíces pigmentados de Chiapas, características físicas, contenido de antocianinas y valor nutraceútico. Rev. Fitotec. Mex. 35:33-41.

Salinas-Moreno Y, G Salas-Sánchez, D Rubio-Hernández, N RamosLobato (2005) Characterization of anthocyanin extracts from maize kernels. J. Chromat. Sci. 43:483-487.

Salinas M Y, H M Soto, B F Martínez, H V González, P R Ortega (1999) Análisis de antocianinas en maíces de grano azul y rojo provenientes de cuatro razas. Rev. Fitotec. Mex. 22:161-174.

Salinas-Moreno Y, J J Pérez-Alonso, G Vázquez-Carrillo, F AragónCuevas, G A Velázquez-Cardelas (2012a) Antocianinas y actividad antioxidante en maíces (Zea mays L.) de las razas Chalqueño, Elotes Cónicos y Bolita. Agrociencia 47:815-825. 
Sánchez J J, M M Goodman, C W Stuber (2000) Isozymatic and morphological diversity in the races of maize of Mexico. Econ. Bot. 54:43-59.

Straus J (1959) Anthocyanin synthesis in corn endosperm tissue culture. I: Identity of the pigments and general factors. Plant Physiol. 536-540.

Styles E D, O Ceska (1972) Flavonoid pigments in genetic strains of maize. Phytochemistry 11:3019-3021.

Wellhausen E J, L M Roberts, E Hernández X, P C Mangelsdorf (1951) Razas de Maíz en México. Su Origen, Características y Distribución. In: Xolocotzia, Obras de Efraím Hernández Xolocotzi. Rev. Geogr. Agríc. Tomo II, 1987. pp:609-732.

Yang S, W Shai (2010) Identification and antioxidant activity of anthocyanins extracted from the seed and cob of purple corn (Zea mays L.). Innov. Food Sci. Emerg. Techn. 11:169-176.
Zhao X, C Zhang, C Guigas, Y Ma, M Corrales, B Tauscher, X Hu (2009) Composition, antimicrobial activity and antiproliferative capacity of anthocyanin extracts of purple corn (Zea mays L.) from China. Eur. Food Res. Technol. 228:759-765.

Zilic S, A Serpen, G Akillioglu, V Gökmen, J Vancetovic (2012) Phenolic compounds, carotenoids, anthocyanins, and antioxidant capacity of colored maize (Zea mays L.) kernels. J. Agric. Food Chem. 60:1224-1231.

Zhu Y, W Ling, H Guo, F Song, Q Ye, T Zou, D Li, Y Zhang, G Li, Y Xiao, F Liu, Z Li, Z Shi, Y Zhang (2013) Anti-inflammatory effect of purified dietary anthocyanins in adult with hypercholesterolemia: A randomized controlled trial. Nutr. Metab. Cardiovas. 23:842-849. 